\title{
Double optical spring enhancement for gravitational-wave detectors
}

\author{
Henning Rehbein, ${ }^{1}$ Helge Müller-Ebhardt, ${ }^{1}$ Kentaro Somiya, ${ }^{2,3}$ Stefan L. Danilishin, ${ }^{1,4}$ Roman Schnabel, ${ }^{1}$ \\ Karsten Danzmann, ${ }^{1}$ and Yanbei Chen ${ }^{2,3}$ \\ ${ }^{1}$ Max-Planck-Institut für Gravitationsphysik (Albert-Einstein-Institut), Institut für Gravitationsphysik, Leibniz Universität Hannover, \\ Callinstrasse 38, 30167 Hannover, Germany \\ ${ }^{2}$ California Institute of Techology, Theoretical Astrophysics 130-33, Pasadena, California 91125, USA \\ ${ }^{3}$ Max-Planck-Institut für Gravitationsphysik (Albert-Einstein-Institut), Am Mühlenberg 1, 14476 Potsdam, Germany \\ ${ }^{4}$ Department of Physics, Moscow State University, Moscow 119992, Russia \\ (Received 20 May 2008; published 16 September 2008)
}

\begin{abstract}
Currently planned second-generation gravitational-wave laser interferometers such as Advanced LIGO exploit the extensively investigated signal-recycling technique. Candidate Advanced LIGO configurations are usually designed to have two resonances within the detection band, around which the sensitivity is enhanced: a stable optical resonance and an unstable optomechanical resonance-which is upshifted from the pendulum frequency due to the so-called optical-spring effect. As an alternative to a feedback control system, we propose an all-optical stabilization scheme, in which a second optical spring is employed, and the test mass is trapped by a stable ponderomotive potential well induced by two carrier light fields whose detunings have opposite signs. The double optical spring also brings additional flexibility in reshaping the noise spectral density and optimizing toward specific gravitational-wave sources. The presented scheme can be extended easily to a multi-optical-spring system that allows further optimization.
\end{abstract}

DOI: 10.1103/PhysRevD.78.062003

PACS numbers: 04.80.Nn, 03.65.Ta, 42.50.Dv, 42.50.Lc

\section{INTRODUCTION}

The large-scale laser interferometers LIGO [1], VIRGO [2], GEO [3] and TAMA [4] represent the first generation of interferometric gravitational-wave $(\mathrm{GW})$ detectors (interferometers for short). Next-generation interferometers, such as Advanced LIGO [5], plan to use the so-called detuned signal-recycling (SR) technique-in which an additional mirror is placed behind the dark port of a Michelson interferometer in order to modify the optical resonance structure of the interferometer: depending on the location and the reflectivity of this signal-recycling mirror, the eigenfrequency and quality factor of the optical resonance can be adjusted [6-8]. As shown theoretically by Buonanno and Chen [9-11] and experimentally by Somiya et al. [12] and Miyakawa et al. [13], detuned signal recycling makes the power inside the interferometer dependent on the motion of the mirrors, creating an optical spring. With optical power as high as planned for secondgeneration interferometers, detuned signal-recycling interferometers are characterized by two resonances within the detection band. One resonance is optical in nature, while the other one is due to the optical spring: the eigenfrequency of the test masses can be shifted from that of the pendulum up into the detection band. The general principle underlying the optical-spring effect in signal-recycling interferometers is identical to that explained by Braginsky and Khalili [14] for a single detuned cavity (cf. [11]), which had been employed in their proposal of the optical bar detection scheme [15].

One concern that arises with the use of the optical spring is that it always causes instability: depending on the sign of the detuning, the optical force either brings antidamping or creates an antispring. Buonanno and Chen have shown in Ref. [10] that one can cope with the instability by incorporating a linear feedback control which ideally would not modify the noise spectral density of the GW detector. In practice, however, the need for control inside the detection band can cause undesirable complexity in the control system or additional classical noise.

Here we propose an alternative way to suppress the instability, by injecting a second carrier field from the bright port (cf. Fig. 1). We shall assume these carriers have different polarizations (as in Ref. [16]), so that there is no direct coupling between the two fields (although they both directly couple to the mirrors). Differently from Ref. [16], in which the second carrier does not enter the arm cavity at all, in this proposal, the second carrier resonates in the arm cavity but is subject to different SR detuning and SR mirror reflectivity. Nevertheless, similarly to Ref. [16], a homodyne detection must be performed at the dark port around each of the two carriers, with the two outputs combined with appropriate filters. The main purpose of this second carrier is to create a second optical spring that forms a stable optical spring together with the first one - even though each individual optical spring, acting alone, would be unstable. Such a stable, double optical spring (DOS) is possible at least in two ways. The first way (weak stabilization) relies on the observation that the ratio between the optical-spring constant's real part (strength of spring/antispring) and its imaginary part (strength of damping/antidamping) depends on the detuning frequency of the carrier-the detunings of the two carriers can be arranged such that the first one has a stronger spring and a weaker antidamping, while the second one has a weaker 


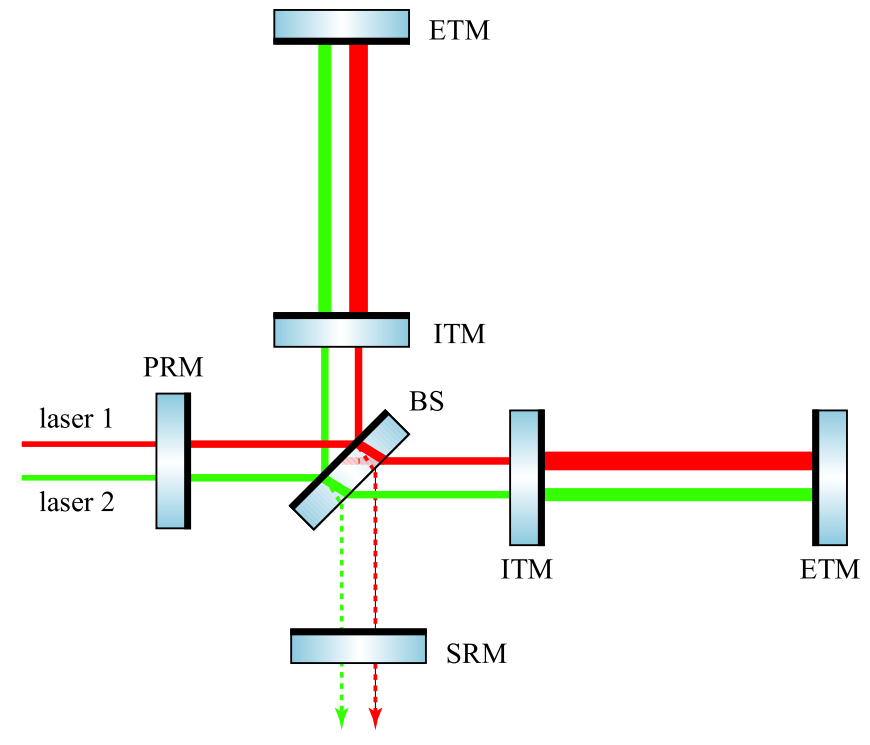

FIG. 1 (color online). Schematic plot of a power- and signalrecycled Michelson interferometer with arm cavities and a double optical spring, which is realized by injecting two carriers with orthogonal polarizations. Both carriers are split at the beam splitter (BS) and transmitted through the ITMs into the arm cavities consisting of ITMs and the end mirrors (ETMs); they both resonate in the arm cavity. Two additional cavities are realized by the SRM and the power-recycling mirror (PRM).

antispring and a stronger damping. In this case, stabilization can be achieved with a weak second carrier, which does not modify the sensitivity of the interferometer by much. The second way (annihilation) requires the two carriers to have equal power and exactly opposite detunings, such that their optical springs exactly cancel each other, and the test masses' differential motion behaves as a free mass again. Interestingly, this configuration gives exactly the noise spectrum that had been expected by the GW community [17] at the time an interferometer with detuned signal recycling was not thoroughly studied, i.e., before Refs. [9-11] were published.

Although straightforward to understand, weak stabilization and annihilation are by no means the only possibilities. In fact, an additional benefit of the DOS technique is that it increases the flexibility in shaping the noise curves: for any specific source, the noise spectrum can be optimized correspondingly over the parameter subspace of the two carriers, subject to the constraint that the resulting dynamics must be stable. In this paper, we will carry out an optimization for neutron-star-neutron-star binary inspirals-using both the current noise budget of Advanced LIGO and a plausible noise budget for interferometers right beyond the second generation. Note that the parameters for the Advanced LIGO configuration as well as for the other single-optical-spring configuration have been obtained by using the same optimization.

The DOS technique can also be used for the stabilization of the optical-spring ponderomotive squeezer, which gen- erates frequency-independent squeezed vacuum below the optical-spring frequency $[18,19]$. The stable optomechanical resonance has already been demonstrated experimentally [20].

This paper is organized as follows: In Sec. II we shall motivate the stabilization process and study the classical dynamics of the double-optical-spring systems. In Sec. III we recall the necessary basics in order to calculate the input-output relation of a DOS interferometer. In Sec. IV different applications of the DOS are discussed, and our scheme is extended to a multi-optical-spring system. In Sec. V we summarize our main conclusions.

\section{CLASSICAL DYNAMICS}

In this section, we consider the classical dynamics of double-optical-spring stabilization. For the mechanical degrees of freedom, we consider only the differential mode

$$
x \equiv x_{\text {antisym }}=\left(x_{\mathrm{ETM}}^{(n)}-x_{\text {ITM }}^{(n)}\right)-\left(x_{\mathrm{ETM}}^{(e)}-x_{\text {ITM }}^{(e)}\right)
$$

between the interferometer's test masses, which is sensed at the dark port by both carriers. Before coupling to the light, the differential mode has an eigenfrequency of $\approx 1 \mathrm{~Hz}$ (that of the suspension system) and effective mass of $m / 4$, where $m$ is the mass of each individual mirror. Since the pendulum frequency is far below the detection band, we will simply treat the mirrors as free masses. For a carrier with angular frequency $\omega_{0}$ that is resonant in the arms, when the mirrors are held fixed, the optical resonant frequency of the differential optical mode (to be precise, the one that is closest to this carrier) is given by $\omega_{0}-\lambda-i \epsilon$, where, in terms of interferometer parameters, $\epsilon$ and $\lambda$ are given by (cf. [11])

$$
\begin{aligned}
& \lambda=\gamma_{o} \frac{2 \rho_{\mathrm{SR}} \sin (2 \phi)}{1+\left(\rho_{\mathrm{SR}}\right)^{2}+2 \rho_{\mathrm{SR}} \cos (2 \phi)}, \\
& \epsilon=\gamma_{o} \frac{1-\left(\rho_{\mathrm{SR}}\right)^{2}}{1+\left(\rho_{\mathrm{SR}}\right)^{2}+2 \rho_{\mathrm{SR}} \cos (2 \phi)},
\end{aligned}
$$

where $\gamma_{0}=T c /(4 L)$ is the half linewidth of the arm cavity ( $T$ the power transmissivity of the input mirrors (ITMs), $c$ the speed of light, and $L$ the arm length), $\rho_{\mathrm{SR}}$ is the amplitude reflectivity of the signal-recycling mirror, and $\phi$ the detuning phase of the carrier in the SR cavity (single trip). In reality, when two carriers are both resonant in the arm cavity, their detuning phases in the signal-recycling cavity must differ by

$$
\Delta \phi=\frac{2 \pi n l_{\mathrm{SR}}}{c}(\Delta \nu)_{\mathrm{FSR}}, \quad n=0, \pm 1, \pm 2, \ldots,
$$

where $(\Delta \nu)_{\mathrm{FSR}}$ is the free spectral range of the arm cavities. This constraint must be taken into account in practical designs of DOS interferometers.

Now with optomechanical coupling, let us first consider a single detuned carrier. Treating the mirrors as free masses 
to start with, the classical equation of motion of the differential mode can be written in frequency domain as

$$
-\frac{m}{4} \Omega^{2} x(\Omega)=-K_{\mathrm{os}}(\Omega) x(\Omega)+F_{\text {ext }},
$$

where $m / 4$ is the reduced mass and $F_{\text {ext }}$ is any external classical force. The frequency-dependent optical-spring constant is given by [11]

$$
K_{\mathrm{os}}=-\frac{m \theta}{4} \frac{\lambda}{(\Omega-\lambda+\mathrm{i} \epsilon)(\Omega+\lambda+\mathrm{i} \epsilon)},
$$

where $\theta$ is given by

$$
\theta=\frac{8 P \omega_{0}}{m L c}
$$

with $P$ the carrier's circulating power in the arms. Note that $\theta$ has units of $\mathrm{Hz}^{3}$.

Before treating a full-power interferometer, it is instructive to first draw our attention to a weakly coupled regime, assuming that optical frequency scales (effective detuning $\lambda$ and effective bandwidth $\epsilon$ ) are much larger than the resulting optomechanical resonant frequency. In the weakly coupled regime, one can expand the optical-spring constant (cf., e.g., Ref. [10]) as

$$
K_{\mathrm{os}}(\Omega) \approx \frac{m \theta \lambda}{4\left(\epsilon^{2}+\lambda^{2}\right)}\left(1+\mathrm{i} \frac{2 \epsilon \Omega}{\left(\epsilon^{2}+\lambda^{2}\right)}\right) \equiv K-\mathrm{i} \Omega \Gamma,
$$

where $K$ and $\Gamma$ are both real constants. Analogous to a mechanical spring, $K$ describes the restoring force while $\Gamma$ denotes the damping. Real and imaginary parts of the optical-spring constant are proportional to $\theta$, which is in turn proportional to the carrier power $P$. Inserting Eq. (8) into Eq. (5), stability requires

$$
K>0 \text { and } \Gamma>0,
$$

or basically positive spring constant and positive damping.

From Eq. (8), it is straightforward to deduce that the stability condition (9) can never be fulfilled by a single optical system, since a positive detuning produces always antidamping $(\Gamma<0)$ while a negative detuning always comes along with an antirestoring force $(K<0)$. In Fig. 2, for fixed circulating power and effective linewidth $(\epsilon)$, as the effective detuning $(\lambda)$ shifts from $-\infty$ to $\infty$, we plot the trajectory mapped out by $(K, \Gamma)$. We use two different powers, with outer trajectory corresponding to a higher power. Indeed, these $\infty$-shaped trajectories are confined within quadrants with $K \cdot \Gamma<0$. In case of a double optical spring, each individual spring, as we change its detuning frequency, has its own $\infty$-shaped trajectory. When two optical springs combine, their complex spring constants add up, which can be depicted by a vector addition in Fig. 2. By adjusting the detunings for the first and second carriers it is possible to find many stable compositions, with one of them depicted in Fig. 2. In this configuration, a

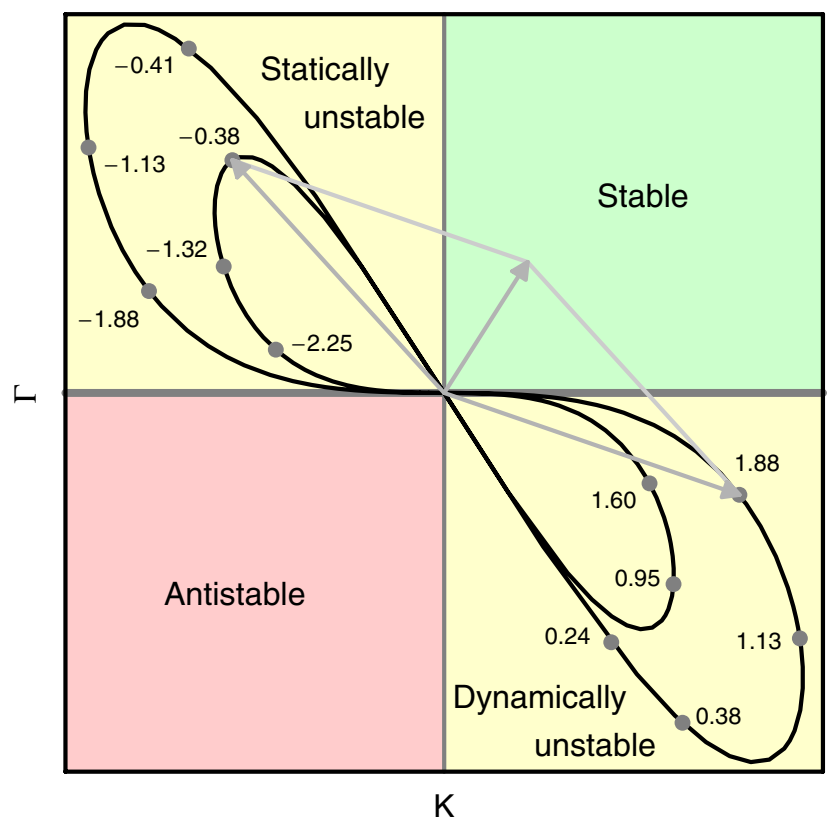

FIG. 2 (color online). Real and imaginary parts of the spring constant $K_{\mathrm{os}}$ in the weakly coupled regime [Eq. (8)]. For each trajectory the circulating power and bandwidth are fixed to a certain value while detuning varies from $-\infty$ to $\infty$. Outer trajectory corresponds to a higher circulating power. Example values of $\lambda / \epsilon$ are marked on the trajectories.

relatively strong optical spring is stabilized by a relatively weak antispring generated by a lower power. This is possible because the stronger optical spring is generated by a carrier with a relatively high optical quality factor $|\lambda / \epsilon|$, which tends to yield a stronger restoring (or antirestoring) than damping (or antidamping), while the weak antispring is generated by a carrier with a low optical quality factor, which tends to yield a stronger damping (or antidamping) than restoring (or antirestoring). A lower optical power of the second carrier allows the damping of the second spring to match that of the first one, while makes the antirestoring of the second spring much weaker than the restoring of the first spring. Mathematically, this weak stabilization can be summarized as

$$
\frac{\left|K^{(1)}\right|}{\left|\Gamma^{(1)}\right|} \gg \frac{\left|K^{(2)}\right|}{\left|\Gamma^{(2)}\right|}, \quad\left|\Gamma^{(1)}\right| \sim\left|\Gamma^{(2)}\right| \Rightarrow\left|K^{(1)}\right| \gg\left|K^{(2)}\right| .
$$

As another (rather extreme) example of DOS stabilization, we note that, when the two carriers have the same power and bandwidth, but the opposite detuning, their opticalspring constants exactly cancel with each other. In fact, this cancellation, or annihilation, is valid for an arbitrarily strong coupling; cf. Eq. (6). Mathematically, this can be summarized as

$$
K_{\mathrm{os}}(\theta, \epsilon, \lambda ; \Omega)+K_{\mathrm{os}}(\theta, \epsilon,-\lambda ; \Omega)=0 .
$$


As stated before, the stability condition given in Eq. (9) is an approximation valid only in the weakly coupled regime. A more precise statement regarding the stability of the two carrier system is given by the condition that all roots of the characteristic equation

$$
-\frac{m}{4} \Omega^{2}+K_{\mathrm{os}}^{(1)}(\Omega)+K_{\mathrm{os}}^{(2)}(\Omega)=0
$$

must have negative imaginary parts. In Fig. 3, we explore high-power DOS stabilization by tracing the real and imaginary parts of the optomechanical eigenfrequency, obtained numerically solving Eq. (12). We first consider single-optical-spring configurations with power increasing from 0 to $800 \mathrm{KW}$ (from the leftmost dot to the top dot). In this case both the real part of the optomechanical resonant frequency and the antidamping increase. Then we fix the first carrier at $800 \mathrm{~kW}$ and increase the second carrier (up to 8 and $80 \mathrm{~kW}$, respectively, for two different choices of linewidth and detuning), which stabilizes the system by adding damping while only slightly decreases the optomechanical resonant frequency by a few hertz (from the top dot to the two lower-right dots). Here we do not plot the optical resonances, which for the circulating power considered here remain stable.

Before ending this section, we note that, when pendulum frequency is not neglected, there does exist a stable singleoptical-spring regime where an increase in mechanical resonant frequency is associated with an increase in damping. But this requires that the optical frequency scales be

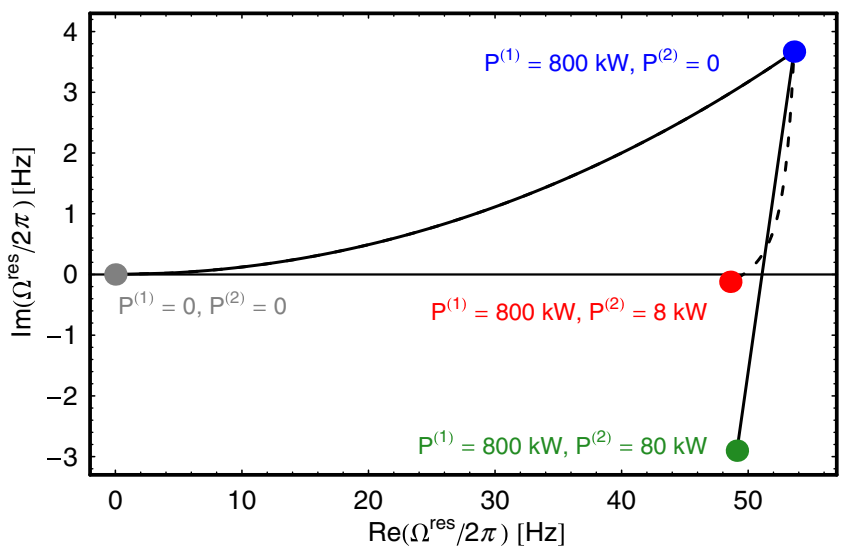

FIG. 3 (color online). Example of the DOS stabilization process, as illustrated by trajectories of the optomechanical resonant frequency in the complex plane [Eq. (12)]. The test masses start off as free masses (gray dot). As the first carrier light $\left(\epsilon^{(1)}=\right.$ $2 \pi 120 \mathrm{~Hz}$ and $\lambda^{(1)}=2 \pi 290 \mathrm{~Hz}$ ) is turned on, it causes an upshift of the mechanical resonant frequency, as well as a mild antidamping. The trajectory ends at the upper right (blue) dot, with $P^{(1)}=800 \mathrm{~kW}$. Subsequently, the second carrier is also turned on, bringing a damping while slightly downshifting the optomechanical resonant frequency. For $\epsilon^{(2)}=2 \pi 5 \mathrm{~Hz}, \lambda^{(2)}=$ $-2 \pi 55 \mathrm{~Hz}$, the trajectory ends at the right (red) dot, with $P^{(2)}=$ $8 \mathrm{~kW}$; while for $\epsilon^{(2)}=2 \pi 60 \mathrm{~Hz}, \lambda^{(2)}=-2 \pi 60 \mathrm{~Hz}$ the trajectory ends at the lower right (green) dot, with $P^{(2)}=80 \mathrm{~kW}$. lower than the pendulum frequency, which is not desirable in the case of GW detectors. Such a regime was experimentally investigated by Schliesser et al. [21].

\section{INPUT-OUTPUT RELATION AND COMBINED NOISE SPECTRAL DENSITY}

In this section, we consider the sensitivity of stable DOS interferometers. Since the two carrier fields are different in polarization and frequency, both fields can be measured independently, and we effectively obtain two interferometers in one scheme, both sensing the same differential mode [Eq. (1)] of mirror motion. The input optical vacuum fluctuations associated with the two carriers, on the other hand, are independent.

Throughout this paper, we will assume that GWs with amplitude $h$ are incident from right above the detector plane, with a polarization that maximizes the response of our $L$-shaped Michelson interferometer. In the following we will list the Heisenberg equations of motion in the frequency domain [9-11,22,23] for the antisymmetric mode of motion of the arm cavity mirrors $\hat{x}$ and for the two measurement outputs $\hat{y}^{(i)}$ :

$$
\begin{aligned}
\hat{x}= & -R_{x x}(\Omega)\left[\hat{F}^{(1)}(\Omega)+\hat{F}^{(2)}(\Omega)+\left(R_{F F}^{(1)}(\Omega)\right.\right. \\
& \left.\left.+R_{F F}^{(2)}(\Omega)\right) \hat{x}\right]+L h+\hat{\xi}_{\text {noise }}, \\
\hat{y}^{(1)}= & \hat{Y}_{1}^{(1)}(\Omega) \sin \zeta^{(1)}+\hat{Y}_{2}^{(1)}(\Omega) \cos \zeta^{(1)} \\
& +\left[R_{Y_{1} F}^{(1)}(\Omega) \sin \zeta^{(1)}+R_{Y_{2} F}^{(1)}(\Omega) \cos \zeta^{(1)}\right] \hat{x}, \\
\hat{y}^{(2)}= & \hat{Y}_{1}^{(2)}(\Omega) \sin \zeta^{(2)}+\hat{Y}_{2}^{(2)}(\Omega) \cos \zeta^{(2)} \\
& +\left[R_{Y_{1} F}^{(2)}(\Omega) \sin \zeta^{(2)}+R_{Y_{2} F}^{(2)}(\Omega) \cos \zeta^{(2)}\right] \hat{x} .
\end{aligned}
$$

Note that we have labeled all quantities with superscripts (1) and (2) for the first carrier and the second carrier, respectively. It was shown in Ref. [24] that the interferometer's output is only marginally influenced by seismic noise, thermal noise and radiation pressure noise introduced at the beam splitter, since the carrier light incident on the beam splitter is weak and the arm cavities prevent fluctuations from building up. The outgoing sideband fields at the dark port around the two different carrier fields are split, and each is sensed independently by a homodyne detection scheme, which measures a certain combination of amplitude and phase quadratures (described by the phases $\zeta^{(1),(2)}$. The operator $\hat{F}^{(i)}$ in Eq. (13) describes the radiation pressure forces which would act on fixed mirrors due to the incoming vacuum fields at the dark port. The operators $\hat{Y}_{j}^{(i)}$ in Eqs. (14) and (15) account for the outgoing fluctuations in the quadratures in case of fixed mirrors. (In the language of Ref. [11], $\hat{F}^{(i)}$ and $\hat{Y}_{j}^{(i)}$ are free quantities.) The operators $\hat{\xi}_{\text {noise }}$ describe the classical displacement noise of the differential mode. The quantity 
$R_{x x} \equiv-4 /\left(m \Omega^{2}\right)$ is the mechanical susceptibility of the differential mode, the susceptibilities $R_{F F}^{(i)}=-K_{\mathrm{os}}^{(i)}$ [cf. Eq. (6)] correspond to the optical-spring constants, and $R_{Y_{i} F}^{(i)}$ are optical transfer functions from the differential mode to the outgoing quadrature fields.

According to Ref. [11], the free quantities $\hat{F}^{(i)}$ and $\hat{Y}_{j}^{(i)}$ are related to incoming amplitude and phase quadratures $\hat{a}_{1}^{(i)}$ and $\hat{a}_{2}^{(i)}$ as

$$
\begin{gathered}
\hat{F}^{(i)}=\sqrt{\frac{\epsilon^{(i)} \theta^{(i)} m \hbar}{2}} \frac{\left(\mathrm{i} \Omega-\epsilon^{(i)}\right) \hat{a}_{1}^{(i)}+\lambda^{(i)} \hat{a}_{2}^{(i)}}{\left(\Omega+\mathrm{i} \epsilon^{(i)}\right)^{2}-\left(\lambda^{(i)}\right)^{2}}, \\
\hat{Y}_{1}^{(i)}=\frac{\left[\left(\lambda^{(i)}\right)^{2}-\left(\epsilon^{(i)}\right)^{2}-\Omega^{2}\right] \hat{a}_{1}^{(i)}+2 \lambda^{(i)} \epsilon^{(i)} \hat{a}_{2}^{(i)}}{\left(\Omega+\mathrm{i} \epsilon^{(i)}\right)^{2}-\left(\lambda^{(i)}\right)^{2}}, \\
\hat{Y}_{2}^{(i)}=\frac{\left[\left(\lambda^{(i)}\right)^{2}-\left(\epsilon^{(i)}\right)^{2}-\Omega^{2}\right] \hat{a}_{2}^{(i)}-2 \lambda^{(i)} \epsilon^{(i)} \hat{a}_{1}^{(i)}}{\left(\Omega+\mathrm{i} \epsilon^{(i)}\right)^{2}-\left(\lambda^{(i)}\right)^{2}} .
\end{gathered}
$$

For vacuum fluctuations, we have

$$
\left\langle\hat{a}_{k}^{(i)}(\Omega)\left(\hat{a}_{l}^{(j)}\right)^{\dagger}\left(\Omega^{\prime}\right)\right\rangle_{\mathrm{sym}}=\pi \delta\left(\Omega-\Omega^{\prime}\right) \delta_{i j} \delta_{k l} .
$$

The optical transfer functions are given by [11]

$$
\begin{gathered}
R_{Y_{1} F}^{(i)}=\sqrt{\frac{\epsilon^{(i)} \theta^{(i)} m}{2 \hbar}} \frac{\lambda^{(i)}}{\left(\Omega+\mathrm{i} \epsilon^{(i)}\right)^{2}-\left(\lambda^{(i)}\right)^{2}}, \\
R_{Y_{2} F}^{(i)}=-\sqrt{\frac{\epsilon^{(i)} \theta^{(i)} m}{2 \hbar}} \frac{\epsilon^{(i)}-\mathrm{i} \Omega}{\left(\Omega+\mathrm{i} \epsilon^{(i)}\right)^{2}-\left(\lambda^{(i)}\right)^{2}} .
\end{gathered}
$$

Finally, the classical noise operator $\hat{\xi}_{\text {noise }}$ satisfies

$$
\left\langle\hat{\xi}_{\text {noise }}(\Omega) \hat{\xi}_{\text {noise }}^{\dagger}\left(\Omega^{\prime}\right)\right\rangle_{\text {sym }}=\pi \delta\left(\Omega-\Omega^{\prime}\right) L^{2} S_{h}^{\mathrm{cl}}(\Omega),
$$

where $S_{h}^{\mathrm{cl}}$ is the classical noise in the gravitational-wave strain.

The outgoing sideband fields at the dark port around the two different carriers are detected independently via homodyne detection. We seek a linear combination of the two measurement output channels, $\hat{y}^{(1)}$ and $\hat{y}^{(2)}$ given in Eqs. (14) and (15):

$$
\hat{y}=K_{1}(\Omega) \hat{y}^{(1)}+K_{2}(\Omega) \hat{y}^{(2)},
$$

which has optimal sensitivity to the GW strain $h$. Namely, one has to minimize the $h$-referred noise spectral density by varying the two filter functions $K_{i}(\Omega)$. The optimal solution can be found by a straightforward calculation described in detail for a general multichannel interferometer in the appendix.

\section{EXAMPLE CONFIGURATIONS}

In this section, different example configurations of a double-optical-spring interferometer are discussed. At first the considerations are restricted to quantum noise only in order to clarify two distinct regimes of our proposed scheme. Subsequently a configuration with a realistic classical noise budget is investigated and optimized for neutron-star-neutron-star binary inspirals. Finally, we lower the classical noise budget and explore whether DOS configurations can take full advantage of this improvement. We are aiming at applying the DOS configuration as an upgrade candidate for the Advanced LIGO detector.

\section{A. Quantum noise examples}

Here we study the quantum noise spectrum of two special regimes of DOS: weak stabilization and annihilation.

Weak stabilization.-In this scenario, we use a relatively weak second carrier to stabilize a typical Advanced LIGO configuration. In Fig. 3, examples were already given, in which a second carrier of 8 and $80 \mathrm{~kW}$, respectively, has been used to stabilize the narrowband mode of Advanced LIGO (cf. Table I), while Fig. 4 provides the region of all possible second carriers, given circulating power of 8,40 and $80 \mathrm{~kW}$.

In Fig. 5, we plot the noise spectra of DOS interferometers that correspond to the two stabilizing configurations in Fig. 3, namely, with the first carrier identical to the carrier of the Advanced LIGO narrowband mode, with resonant frequencies and powers of the second carrier given in Fig. 3, and a phase readout quadrature associated with the second carrier (i.e., $\zeta^{(2)}=0$ ). As we see from Fig. 5, the DOS noise spectra in both cases do not differ much from that of the Advanced LIGO narrowband mode.

Annihilation.-Now we turn to a different situation where both carriers have half the Advanced LIGO circulating power, namely, $400 \mathrm{~kW}$ with the two detunings exactly opposite. In this case the two optical springs cancel each other, and the total effective ponderomotive rigidity vanishes. For Fig. 6 the two detection angles also have opposite signs, while the absolute values of the two detunings (detection angles) agree with the detuning (detection angle) in the case of the conventional Advanced LIGO configurations (cf. Table I). All other parameters are left unchanged.

The sensitivity does not change in the high-frequency regime where shot noise is the limiting factor (cf. Fig. 6). This is because, generally, the noise spectral density of the shot noise remains unchanged under reversing the sign of the detuning and the detection angle. With the optimal filter (cf. Fig. 7), we obtain the same shot noise as the Advanced LIGO configuration, as the total power is conserved. In the low-frequency regime the sensitivity is slightly improved compared to the single-optical-spring interferometer. It is well known that the quantum-noise limited sensitivity of single-optical-spring interferometers at frequencies below the optomechanical resonance is dramatically lower than the sensitivity of non-optical- 
TABLE I. Parameter values for single-optical-spring Advanced LIGO interferometer configurations used throughout the calculations. The narrowband configuration is optimized for NS-NS binaries using the current Advanced LIGO noise budget. For the broadband operational mode, we allow a $10 \%$ decrease in the detectable distance for NS-NS binaries assuming the current Advanced LIGO noise budget, while maximizing the contribution to SNR from frequencies above $500 \mathrm{~Hz}$.

\begin{tabular}{lccc}
\hline \hline Symbol & Physical meaning & AdvLIGO narrowband & AdvLIGO broadband \\
\hline$m$ & Single mirror mass & $40 \mathrm{~kg}$ & $40 \mathrm{~kg}$ \\
$2 \pi c / \omega_{0}^{(1)}$ & Laser wavelength & $1064 \mathrm{~nm}$ & $1064 \mathrm{~nm}$ \\
$P^{(1)}$ & Circulating power & $800 \mathrm{~kW}$ & $800 \mathrm{~kW}$ \\
$L^{(1)}$ & Interferometer arm length & $4 \mathrm{~km}$ & $4 \mathrm{~km}$ \\
$\phi^{(1)}$ & Detuning phase of SR cavity & $2 \pi 0.242$ & $2 \pi 0.247$ \\
$\rho_{\mathrm{SR}}$ & Signal-recycling mirror reflectivity & $\sqrt{0.93}$ & $\sqrt{0.93}$ \\
$\gamma_{o}$ & Cavity half bandwidth & $2 \pi 15 \mathrm{~Hz}$ & $2 \pi 15 \mathrm{~Hz}$ \\
$\zeta^{(1)}$ & Detection angle & $2 \pi 0.347$ & $2 \pi 0.45$ \\
$\epsilon$ & Effective half bandwidth & $2 \pi 120 \mathrm{~Hz}$ & $2 \pi 395 \mathrm{~Hz}$ \\
$\lambda$ & Effective detuning & $2 \pi 290 \mathrm{~Hz}$ & $2 \pi 411 \mathrm{~Hz}$ \\
\hline \hline
\end{tabular}

spring interferometers because the strong restoring force due to the single optical spring suppresses the response of the interferometer's differential mode to GWs. In a doubleoptical-spring interferometer the second carrier usually results in a less rigid or completely canceled effective optical spring. This effect appears in the first example where only a weak second carrier is used (cf. Fig. 5) and becomes more significant here in the case of canceled optical springs (cf. Fig. 6). For intermediate frequencies the sensitivity becomes worse due to the absence of the optomechanical resonance gain. In this regime the output

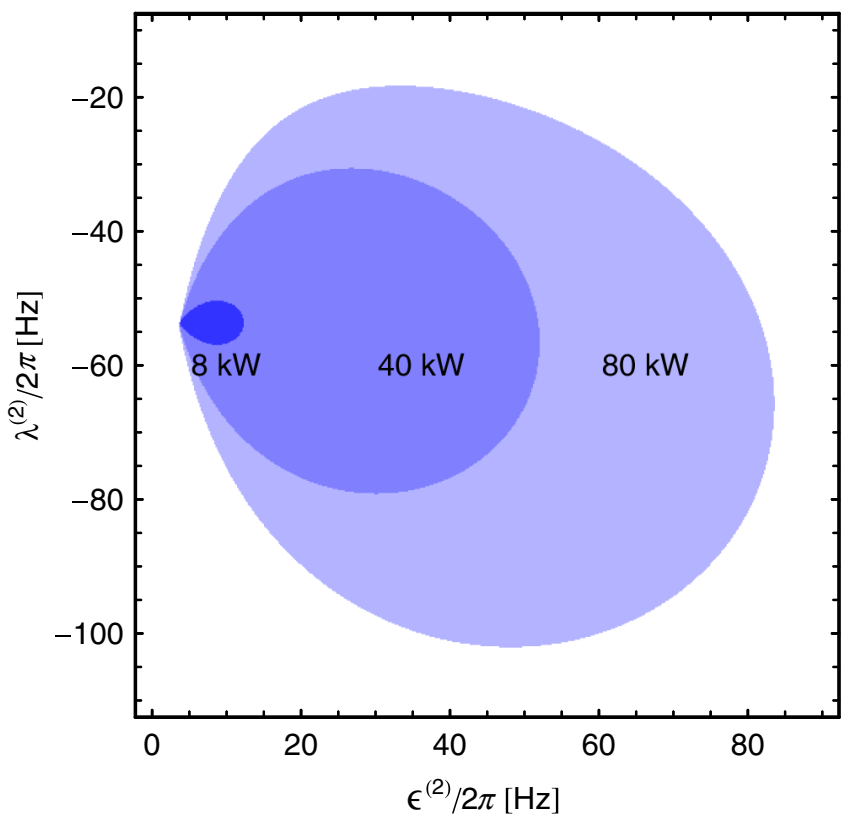

FIG. 4 (color online). Allowable regions for the optical resonances of the second carrier, given a circulating power of 8,40 and $80 \mathrm{~kW}$, in order to stabilize a $800 \mathrm{~kW}$ first carrier that corresponds to the Advanced LIGO narrowband mode (cf. Table I). associated with the carrier having positive detuning usually has more sensitivity than the other one. Therefore, as shown in Fig. 7, the filters make sure that only this output contributes to the total output. Note that when the two outputs are filtered appropriately, the total noise curve can in fact be below the single contributions.

Since the optomechanical resonance disappears completely, the noise spectral density in this configuration is equal to

$$
S_{h}(\Omega)=S_{\text {shot }}(\Omega)+\frac{S_{\mathrm{SQL}}^{2}(\Omega)}{4 S_{\text {shot }}(\Omega)} .
$$

Here $S_{\text {shot }}(\Omega)$ denotes the shot-noise spectral density of a detuned single carrier interferometer with the same total power as in the canceled optical-spring configuration. The

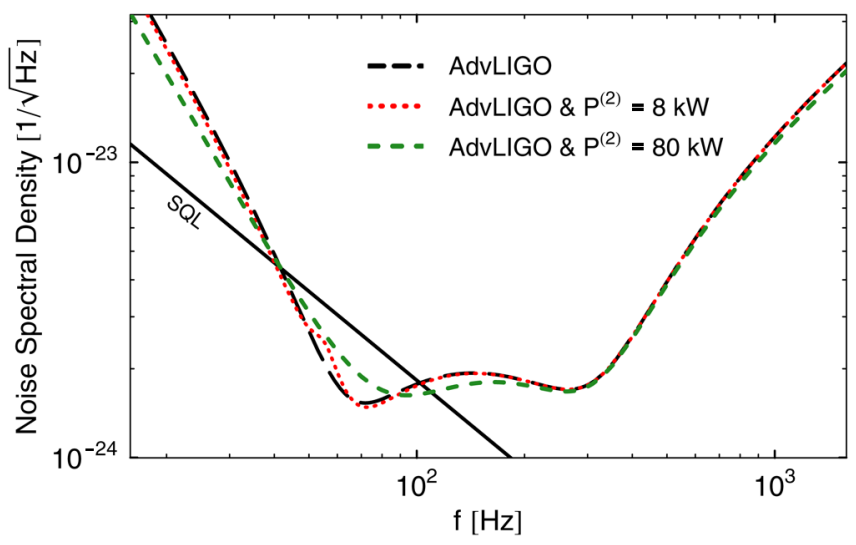

FIG. 5 (color online). Noise spectrum of weak-stabilization DOS configurations, with parameters corresponding to the configuration in Fig. 3. Namely, the first carrier is identical to the carrier in the Advanced LIGO narrowband mode (cf. Table I). Two possible choices for the stabilizing second carrier are (i) $P^{(2)}=8 \mathrm{~kW}, \quad \epsilon^{(2)}=2 \pi 5 \mathrm{~Hz}, \quad \lambda^{(2)}=-2 \pi 55 \mathrm{~Hz}$ and (ii) $P^{(2)}=80 \mathrm{~kW}, \epsilon^{(2)}=2 \pi 60 \mathrm{~Hz}, \lambda^{(2)}=-2 \pi 60 \mathrm{~Hz}$. Phase quadrature detection $\left(\zeta^{(2)}=0\right)$ in both cases. 


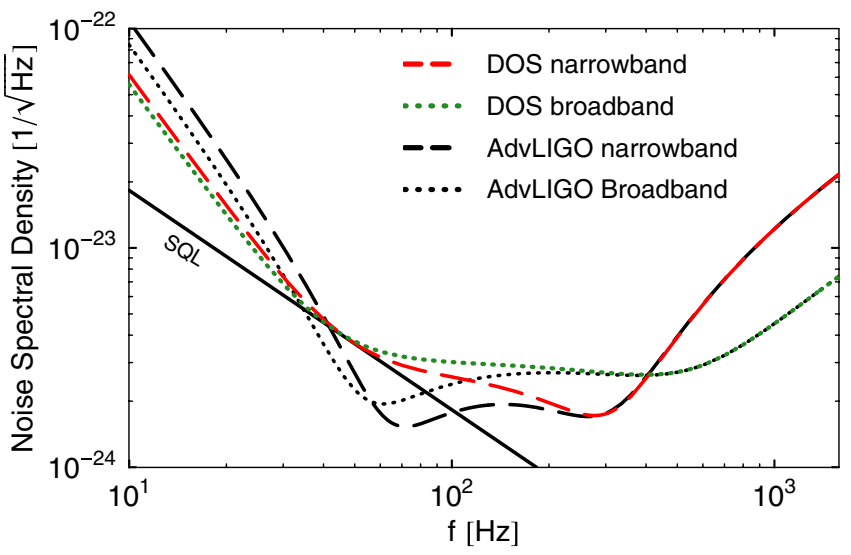

FIG. 6 (color online). Noise spectrum of DOS configurations with canceled optical springs, based on Advanced LIGO narrowband and broadband modes (cf. Table I). The detuning and detection angle of the first carrier are identical to those of Advanced LIGO narrowband mode, while those of the second are opposite. The total power is equally divided into two parts $\left(P^{(1)}=P^{(2)}=400 \mathrm{~kW}\right)$. Other parameters are left unchanged.

free-mass standard quantum limit (SQL) [25] for detecting the gravitational-wave strain $h$ with a Michelson interferometer with arm cavities is given by

$$
S_{\mathrm{SQL}}(\Omega)=\sqrt{\frac{8 \hbar}{m \Omega^{2} L^{2}}} .
$$

\section{B. Optimized configurations with the Advanced LIGO classical noise budget}

In the following we optimize the double-optical-spring interferometer for neutron-star-neutron-star (NS-NS) binary inspirals. For such systems the last stable circular

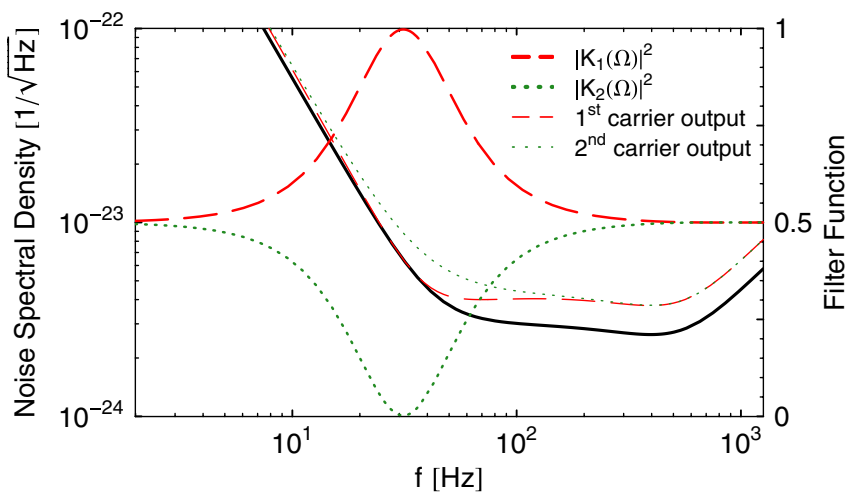

FIG. 7 (color online). Example by means of the broadband configuration (cf. Fig. 6): squared absolute values of filter functions for the two outputs. These curves actually account for how much of the contributions is used-but they cannot show how the correlations between the two outputs have influence on the total output. The contributions correspond to measuring one of the outputs alone without filtering-but still in the presence of both carriers. orbit gives an upper frequency limit of $f_{\max } \approx 1570 \mathrm{~Hz}$ and seismic noise defines a lower bound of $f_{\min } \approx 7 \mathrm{~Hz}$; the signal-to-noise ratio ( $\mathrm{SNR}$ ) in power achievable by optimal filtering, for a source at a given radius, is given by

$$
\mathrm{SNR}^{2} \propto \int_{f_{\min }}^{f_{\max }} \frac{f^{-7 / 3}}{S_{h}(f)} \mathrm{d} f .
$$

Here $S_{h}(f)$ denotes the single-sided noise spectral density of the interferometer. Note that this optimization strategy tends to focus more on the low-frequency regime at the expense of the sensitivity at higher frequencies - due to the rather steep power law of $f^{-7 / 3}$. Since the radius of detectable range is proportional to the SNR at a fixed radius, and the event rate is roughly the cube of the radius of detectable range, the event rate is proportional to $\mathrm{SNR}^{3}$, or

$$
\text { event rate } \propto\left[\int_{f_{\min }}^{f_{\max }} \frac{f^{-7 / 3}}{S_{h}(f)} \mathrm{d} f\right]^{3 / 2}
$$

For the optimization we have taken the current Advanced LIGO classical noise budget into account (as given in Bench [26]): each contribution to the total classical noise budget, i.e., suspension thermal noise, seismic noise, thermal fluctuations in the coating and gravity gradient noise, are presented in Figs. 8 and 9.

It turns out that the second carrier not only helps to stabilize the interferometer but can also improve its sensitivity. We first assume both carriers to have the same SR mirror reflectivity; in this case the gain in NS-NS sensitivity is maximized when the two optical springs totally cancel each other (Fig. 8) at an equal power distribution in the two carrier fields. Figure 8 also shows the contribution of each carrier to the total noise spectral density.

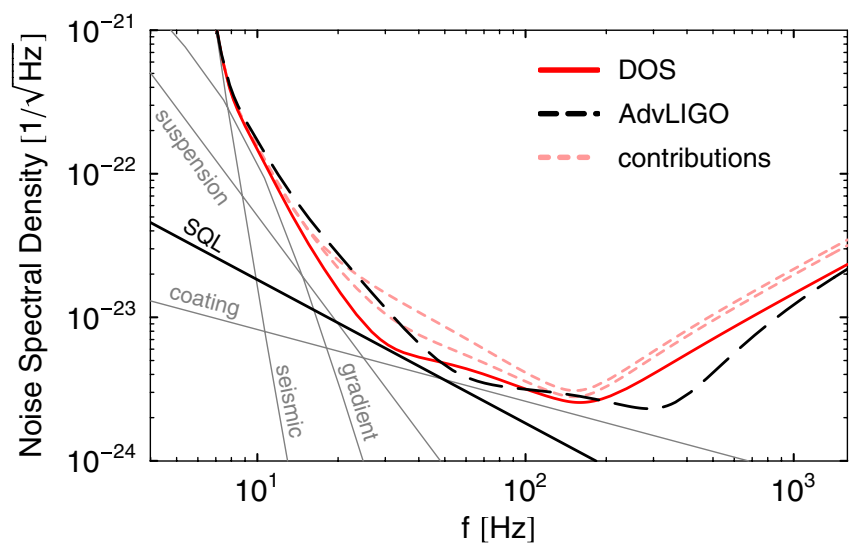

FIG. 8 (color online). Comparison of single-optical-spring and double carrier with canceled optical-spring Advanced LIGO configuration: the same reflectivity of the SR mirror is assumed for both carriers. We obtain $15 \%$ improvement in the event rate for the DOS interferometer using the following parameters: $P^{(1)}=P^{(2)}=400 \mathrm{~kW}, \rho_{\mathrm{SRM}}^{(1)}=\rho_{\mathrm{SRM}}^{(2)}=0.87, \phi^{(1)}=-\phi^{(2)}=$ $2 \pi 0.233, \zeta^{(1)}=2 \pi 0.433$ and $\zeta^{(2)}=0$. 


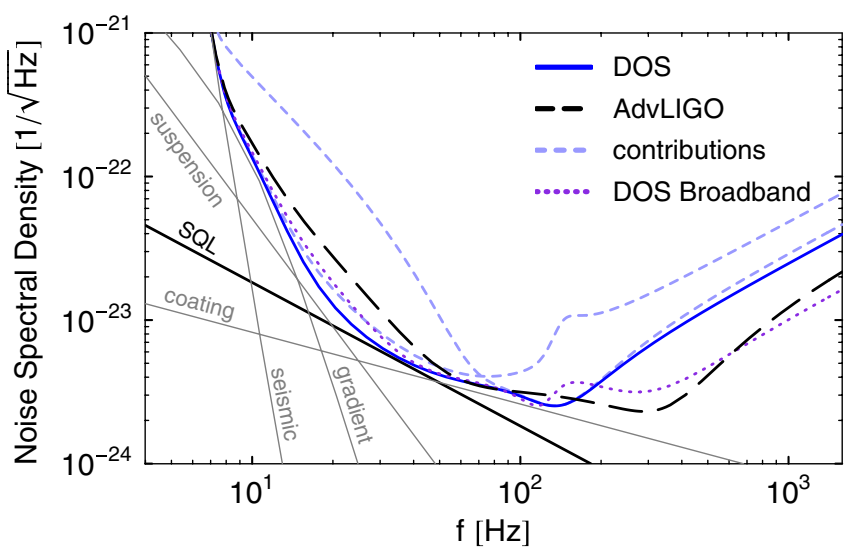

FIG. 9 (color online). Comparison of single- and double-optical-spring Advanced LIGO configurations: different SR mirror reflectivities for first and second carriers are allowed. Parameters are given in detail in the last row of the first block in Table II for the narrowband configuration. For the broadband we have $\lambda^{(1)}=2 \pi 130 \mathrm{~Hz}, \quad \epsilon^{(1)}=2 \pi 5 \mathrm{~Hz}, \quad \zeta^{(1)}=2 \pi 0.01, \quad \lambda^{(2)}=$ $-2 \pi 230 \mathrm{~Hz}, \epsilon^{(2)}=2 \pi 155 \mathrm{~Hz}$ and $\zeta^{(2)}=2 \pi 0.02$. We obtain a $35.5 \%$ improvement in the event rate in the case of the narrowband configuration and $16.5 \%$ for the broadband configuration.

If each carrier senses a different reflectivity of the SR mirror, the interferometer's sensitivity can be improved further. With this additional degree of freedom the situation of canceled optical springs is no longer the optimal choice for NS-NS binaries. Each carrier can be optimized for a different frequency regime such that they complement each other. This is illustrated for an equal distribution of the total power ( $400 \mathrm{~kW}$ for each carrier) in Fig. 9, where one carrier ensures good sensitivity in the low-frequency regime while the other one gives the main contribution at frequencies above the optomechanical resonance as shown by the contribution curves. The noise spectral density is close to the classical noise level at low frequencies, and an improvement in the event rate of $35.5 \%$ can be achieved for NS-NS binaries for which the optimization was performed. As a more general optimization is performed, allowing two carriers to have different powers summing up to $800 \mathrm{~kW}$, it turns out that $P^{(1)}=500 \mathrm{~kW}, P^{(2)}=300 \mathrm{~kW}$ achieves a slightly better event rate improvement of $36.5 \%$. Results for different power distributions are given in Table II.

The sensitivity of all optimized configurations is basically improved at low frequencies at the expense of the high frequencies. Therefore it might be necessary to carry out an additional broadband optimization in order to achieve a better sensitivity in the high-frequency regime. This can be accomplished by first picking out all configurations obeying an event rate which is at least a certain fraction of the optimal event rate for NS-NS binary systems [cf. Eq. (26)]. In a second step these configurations are explored in the high-frequency regime by considering a smaller frequency integration interval $[500 \mathrm{~Hz}, 1570 \mathrm{~Hz}]$ and selecting the optimal signal-to-noise ratio on this interval. For the $\left(P^{(1)}=P^{(2)}=400 \mathrm{~kW}\right)$ configuration as an example, we can achieve a sensitivity comparable to Advanced LIGO on the $[500 \mathrm{~Hz}, 1570 \mathrm{~Hz}]$ frequency band while maintaining an improvement in the event rate (integrating again from $f_{\min }$ ) of $16.5 \%$ compared to Advanced LIGO (cf. Fig. 9).

After adding up all classical noise contribution shown in Figs. 8 and 9 it turns out that the noise spectral density of the double-optical-spring configuration-contrary to the single optical spring in Advanced LIGO_almost follows the borderline set by the classical noise in the lowfrequency regime. Let us write

TABLE II. Parameters for double-optical-spring scheme optimized for NS-NS binary systems. The total power is fixed to $800 \mathrm{~kW}$. The last column gives the improvement in the event rate for our proposed scheme compared to an optimized Advanced LIGO configuration provided in the first row. For the upper part we adopted the current Advanced LIGO noise budget, and for the lower part the gravity gradient noise and the suspension thermal noise are reduced by a factor of 10 and the coating thermal by a factor of 3 .

\begin{tabular}{|c|c|c|c|c|c|c|c|c|c|}
\hline & $\begin{array}{c}P^{(1)} \\
\text { in } \mathrm{kW}\end{array}$ & $\begin{array}{c}P^{(2)} \\
\text { in } \mathrm{kW}\end{array}$ & $\begin{array}{c}\lambda^{(1)} \\
\text { in } \mathrm{Hz}\end{array}$ & $\begin{array}{c}\lambda^{(2)} \\
\text { in } \mathrm{Hz}\end{array}$ & $\begin{array}{c}\boldsymbol{\epsilon}^{(1)} \\
\text { in } \mathrm{Hz}\end{array}$ & $\begin{array}{c}\boldsymbol{\epsilon}^{(2)} \\
\text { in } \mathrm{Hz}\end{array}$ & $\begin{array}{c}\zeta^{(1)} \\
\text { in radians }\end{array}$ & $\begin{array}{c}\zeta^{(2)} \\
\text { in radians }\end{array}$ & Improvement \\
\hline \multirow[t]{7}{*}{ High classical noise } & 800 & 0 & $2 \pi 290$ & $\cdots$ & $2 \pi 120$ & $\ldots$ & $2 \pi 0.347$ & $\ldots$ & $\ldots$ \\
\hline & 750 & 50 & $2 \pi 190$ & $-2 \pi 70$ & $2 \pi 50$ & $2 \pi 20$ & $2 \pi 0.125$ & $2 \pi 0.075$ & $7 \%$ \\
\hline & 700 & 100 & $2 \pi 190$ & $-2 \pi 50$ & $2 \pi 40$ & $2 \pi 30$ & $2 \pi 0.0625$ & $2 \pi 0.475$ & $22.5 \%$ \\
\hline & 600 & 200 & $2 \pi 180$ & $-2 \pi 40$ & $2 \pi 30$ & $2 \pi 50$ & $2 \pi 0.0125$ & $2 \pi 0.3875$ & $34.5 \%$ \\
\hline & 500 & 300 & $2 \pi 150$ & $-2 \pi 50$ & $2 \pi 45$ & $2 \pi 60$ & $2 \pi 0.0125$ & $2 \pi 0.375$ & $36.5 \%$ \\
\hline & 450 & 350 & $2 \pi 160$ & $-2 \pi 10$ & $2 \pi 30$ & $2 \pi 40$ & $2 \pi 0$ & $2 \pi 0.3$ & $35.5 \%$ \\
\hline & 400 & 400 & $2 \pi 140$ & $-2 \pi 20$ & $2 \pi 20$ & $2 \pi 55$ & $2 \pi 0.01$ & $2 \pi 0.31$ & $35.5 \%$ \\
\hline \multirow[t]{6}{*}{ Low classical noise } & 800 & 0 & $2 \pi 170$ & $\ldots$ & $2 \pi 10$ & $\ldots$ & $2 \pi 0.263$ & $\ldots$ & $\ldots$ \\
\hline & 700 & 100 & $2 \pi 165$ & $-2 \pi 75$ & $2 \pi 5$ & $2 \pi 25$ & $2 \pi 0.48$ & $2 \pi 0.02$ & $32 \%$ \\
\hline & 600 & 200 & $2 \pi 150$ & $-2 \pi 20$ & $2 \pi 5$ & $2 \pi 45$ & $2 \pi 0.01$ & $2 \pi 0.36$ & $52 \%$ \\
\hline & 500 & 300 & $2 \pi 140$ & $-2 \pi 20$ & $2 \pi 5$ & $2 \pi 45$ & $2 \pi 0.03$ & $2 \pi 0.33$ & $74.5 \%$ \\
\hline & 450 & 350 & $2 \pi 135$ & $-2 \pi 25$ & $2 \pi 5$ & $2 \pi 50$ & $2 \pi 0.01$ & $2 \pi 0.33$ & $83.5 \%$ \\
\hline & 400 & 400 & $2 \pi 127$ & $-2 \pi 11$ & $2 \pi 4$ & $2 \pi 46$ & $2 \pi 0.01$ & $2 \pi 0.3$ & $110 \%$ \\
\hline
\end{tabular}




$$
\begin{aligned}
\eta & \equiv \int_{f_{\min }}^{150 \mathrm{~Hz}} \frac{f^{-7 / 3}}{S_{h}(f)} \mathrm{d} f / \int_{f_{\min }}^{150 \mathrm{~Hz}} \frac{f^{-7 / 3}}{S_{h}^{\mathrm{cl}}(f)} \mathrm{d} f \equiv \frac{\bar{S}_{h}^{\mathrm{cl}}}{\bar{S}_{h}} \\
& =\frac{\bar{S}_{h}^{\mathrm{cl}}}{\bar{S}_{h}^{\mathrm{cl}}+\bar{S}_{h}^{\mathrm{q}}}
\end{aligned}
$$

where $\bar{S}_{h}^{\mathrm{cl}}, \bar{S}_{h}^{\mathrm{q}}$ and $\bar{S}_{h}$ are weighted averages of classical, quantum, and total noise spectrum in the low-frequency band, respectively. For the $P^{(1)}=P^{(2)}=400 \mathrm{~kW}$ configuration (cf. Fig. 9) we obtain

$$
\eta \approx 0.81 \text {. }
$$

This indicates that, at low frequencies (i.e., below $150 \mathrm{~Hz}$ ), the quantum noise is already a small fraction of the total noise; improving quantum noise further does not significantly improve sensitivity. Qualitatively, starting with a level of $\eta=0.81$, further lowering $\bar{S}_{h}^{\mathrm{q}}$ by a factor of 2 only improves $\eta$ from 0.81 to 0.88 , which yields a $14 \%$ increase in event rate.

\section{Optimized configurations with a classical noise budget beyond Advanced LIGO}

It is likely that technical improvements will reduce the classical noise floor in the future. In order to explore the potential of our proposed configuration to increase the quantum limited sensitivity by reshaping the noise curves in an optimal way, we analyze the performance for a reduced classical noise budget. For instance, the gravity gradient noise is a limiting factor at lower frequencies. As suggested in Ref. [27] this effect can be removed from the recorded data by performing an independent measurement of the ground's density fluctuations near each test mass. We assume it to be 1/10 (in amplitude) the current estimation for Advanced LIGO [28]. Another limiting factor is given by the thermal noise in the suspension system and mirrors. We assume that the suspension thermal noise can be lowered by a factor of 10 in amplitude, while the internal thermal noise of mirrors can be lowered by a factor of 3 in amplitude [28]. Such improvements may possibly be realized by (i) optimizing the design of the mirror coating structure and suspension wires, (ii) improving mechanical quality factors of the mirror coating, substrate and suspension materials, and (iii) applying cryogenic techniques [17,29-32].

Now we optimize the usual single-optical-spring configuration as well as our proposed double-optical-spring layout for this modified noise budget. Only now does the potential of the two carrier interferometer become apparent: for the new classical noise budget, the single-opticalspring configuration can improve the event rate for NS-NS binary inspirals only by $61 \%$. But the DOS configuration can do $238 \%$. This corresponds to an improvement in the event rate of the DOS compared to the optimized (with respect to the new classical noise budget) single-opticalspring configuration of $110 \%$ (cf. Table II).

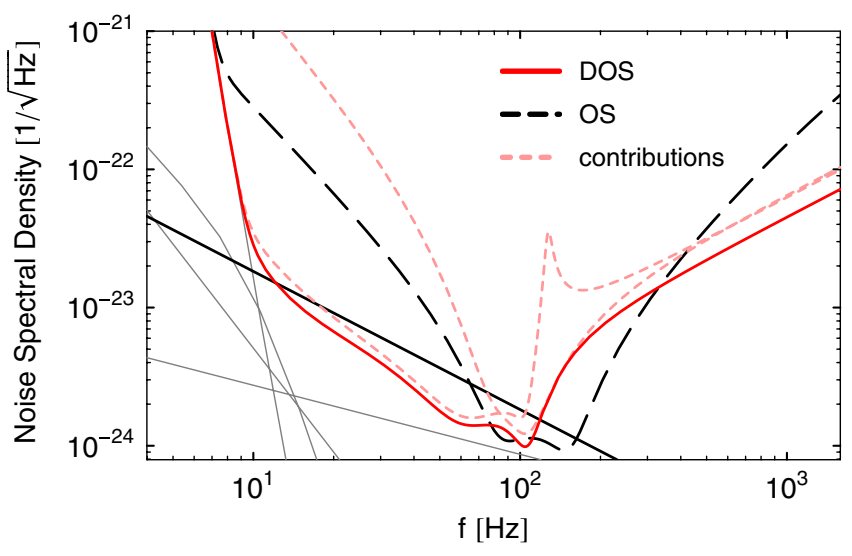

FIG. 10 (color online). Compared to Fig. 8 suspension thermal noise and gravity gradient noise are lowered by a factor of 10 and coating thermal noise by a factor of 3 . The DOS configuration as well as the single-optical-spring configuration are both optimized with respect to the new noise budget. An improvement in the event rate of $238 \%$ can be achieved by DOS configurations, compared with $61 \%$ of single-optical-spring configurations. The following parameters were used: $P^{(1)}=$ $P^{(2)}=400 \mathrm{~kW}, \lambda^{(1)}=2 \pi 130 \mathrm{~Hz}, \lambda^{(2)}=-2 \pi 10 \mathrm{~Hz}, \epsilon^{(1)}=$ $2 \pi 5 \mathrm{~Hz}, \epsilon^{(2)}=2 \pi 45 \mathrm{~Hz}, \zeta^{(1)}=2 \pi 0.46, \zeta^{(2)}=2 \pi 0.3$. For single-optical-spring optimization, parameters are given in the first row of the second block in Table II.

Such a situation is presented in Fig. 10. The big gap between the single-optical-spring total noise and the classical noise budget verifies that the single-optical-spring Advanced LIGO configuration has not been limited by classical noise at low frequencies. While this gap can be partially filled by the double-optical-spring configuration, there is still room for further improvement. For the configuration used in Fig. 10 we evaluate

$$
\eta \approx 0.3 \text {. }
$$

In this case, lowering the quantum noise by a factor of 2 improves the event rate by $69 \%$. One possibility of further improvement would be to inject even more than two carriers and combine the corresponding output channels (cf. the appendix).

\section{CONCLUSION}

While the concept of a stable DOS has motivated experiments such as the one already carried out by Corbitt et al. [20], in this paper, we have theoretically investigated the benefit of a DOS configuration for second-generation gravitational-wave detectors, in particular, in a follow-up experiment to Advanced LIGO, possibly in combination with other existing schemes, e.g., the local readout scheme proposed in Ref. [16], as well as the injection of squeezed vacuum [33-37].

In the DOS configuration, a second laser beam is injected into signal-recycling interferometers at the bright port which is, as the first carrier, resonant in the arm 
cavities and is also detuned in the signal-recycling cavity. The two outputs are optimally filtered and combined. By choosing appropriate detunings of the signal-recycling cavity and homodyne detection angles, it is possible to achieve a stable double optical spring while additionally improving the sensitivity.

Taking into account the current classical noise budget estimation for the Advanced LIGO detector, as well as constraints on optical power, we have performed an optimization of our double-carrier scheme towards the detection of compact binary inspirals specifying to neutron stars. The DOS allows a $36 \%$ improvement in the event rate, and we have shown that further improvement in the event rate will largely be limited by classical noise. When considering a more optimistic classical noise budget, DOS interferometers are much more capable than single-opticalspring interferometers in taking advantage of this improvement: compared with $61 \%$ improvement in the event rate achievable by single-optical-spring configurations, the DOS allows $238 \%$. Nevertheless the reduced classical noise level leaves further room beyond DOS, which can be exploited by employing more than two carrier fields.

\section{ACKNOWLEDGMENTS}

We thank S. Waldman and K. Strain as well as T. Corbitt, N. Mavalvala and C. Wipf for very useful discussions. We thank R. Adhikari for suggesting the level of reduction of classical noise. Research of H. M.-E., K. S. and Y.C. is supported by the Alexander von Humboldt Foundation's Sofja Kovalevskaja Program. Y.C. is also supported by NSF Grants No. PHY-0653653 and No. PHY-0601459, as well as the David and Barbara Groce startup fund at Caltech. Research of S. L.D. is also supported by the Alexander von Humboldt Foundation. Research of H. R. and R.S. is supported by the Deutsche Forschungsgemeinschaft through the SFB No. 407.

\section{APPENDIX: OPTIMAL OUTPUT CHANNEL FOR INTERFEROMETERS WITH MULTIPLE CARRIERS}

Here we analyze the optimal way of combining the output channels obtained from homodyne detections at the dark port of a multicarrier interferometer. The general equation of motion for the mirror position in case of $n$ carriers reads [cf. Eq. (13)]:

$$
\hat{x}=-R_{x x}(\Omega) \sum_{i=1}^{n}\left[\hat{F}^{(i)}+R_{F F}^{(i)}(\Omega) \hat{x}\right]+L h+\hat{\xi}_{\text {noise }}
$$

The output corresponding to each of the $n$ carrier fields is given by [cf. Eqs. (14) and (15)]

$$
\begin{aligned}
\hat{y}^{(i)}(\Omega)= & \hat{Y}_{1}^{(i)}(\Omega) \sin \zeta^{(i)}+\hat{Y}_{2}^{(i)}(\Omega) \cos \zeta^{(i)} \\
& +\left[R_{Y_{1} F}^{(i)}(\Omega) \sin \zeta^{(i)}+R_{Y_{2} F}^{(i)}(\Omega) \cos \zeta^{(i)}\right] \hat{x} \\
\equiv & \vec{n}_{i}^{T} \vec{\nu}+s_{i} h,
\end{aligned}
$$

where $\vec{\nu}$ is a vector with $2 n+1$ entries which account for $2 n$ quadrature operators and one operator modeling the classical noise. Here $\vec{n}_{i}$ describes the noise transfer functions from the noise channels $\vec{\nu}$ into the output channels, and $s_{i}$ accounts for the signal-transfer functions. The combined output is given by

$$
\hat{y}(\Omega)=\sum_{i=1}^{n} K_{i}(\Omega) \hat{y}^{(i)}(\Omega),
$$

and one has to identify $n$ optimal filter functions which minimize the signal-referred noise spectral density of $\hat{y}$ :

$$
S_{h}(\Omega)=\frac{\sum_{i, j=1}^{n}(\mathbf{N})_{i j} K_{i} K_{j}^{*}}{\sum_{i, j=1}^{n}(\mathbf{S})_{i j} K_{i} K_{j}^{*}},
$$

with

$$
(\mathbf{S})_{i j}=s_{i} s_{j}^{*}
$$

and

$$
(\mathbf{N})_{i j}=\sum_{s, k=1}^{2 n+1}\left(\vec{n}_{i}^{T}\right)_{s} S_{\nu_{s} \nu_{k}}\left(\vec{n}_{j}^{\dagger}\right)_{k}
$$

Here $S_{\nu_{s} \nu_{k}}$ is the cross spectral density between $\nu_{s}$ and $\nu_{k}$, with

$$
\left\langle\nu_{s}(\Omega) \nu_{k}^{\dagger}\left(\Omega^{\prime}\right)\right\rangle=2 \pi \delta\left(\Omega-\Omega^{\prime}\right) \frac{S_{\nu_{s} \nu_{k}}(\Omega)}{2} .
$$

The inverse of the largest eigenvalue of the $n \times n$ matrix

$$
\mathbf{M}=\mathbf{N}^{-1} \cdot \mathbf{S}
$$

provides the resulting minimum noise, and the corresponding eigenvector gives the $n$ optimal filter function $K_{i}$.
[1] D. Shoemaker et al. (LIGO Scientific Collaboration), Nucl. Instrum. Methods Phys. Res., Sect. A 517, 154 (2004).

[2] L.D. Fiore et al. (VIRGO Collaboration), Classical Quantum Gravity 19, 1421 (2002).
[3] B. Willke et al., Classical Quantum Gravity 19, 1377 (2002).

[4] M. Ando et al. (TAMA Collaboration), Phys. Rev. Lett. 86, 3950 (2001).

[5] Report No. ligo-060056-07-m, 2006 (http://www.ligo. 
caltech.edu/docs/M/M060056-07/M060056-07.pdf).

[6] B. Meers, Phys. Rev. D 38, 2317 (1988).

[7] J. Vinet, B. Meers, C. Man, and A. Brillet, Phys. Rev. D 38, 433 (1988).

[8] G. Heinzel, K. A. Strain, J. Mizuno, K. D. Skeldon, B. Willke, W. Winkler, R. Schilling, A. Rüdiger, and K. Danzmann, Phys. Rev. Lett. 81, 5493 (1998).

[9] A. Buonanno and Y. Chen, Phys. Rev. D 64, 042006 (2001).

[10] A. Buonanno and Y. Chen, Phys. Rev. D 65, 042001 (2002).

[11] A. Buonanno and Y. Chen, Phys. Rev. D 67, 062002 (2003).

[12] K. Somiya, P. Beyersdorf, K. Arai, S. Sato, S. Kawamura, O. Miyakawa, F. Kawazoe, S. Sakata, A. Sekido, and N. Mio, Appl. Opt. 44, 3179 (2005).

[13] O. Miyakawa et al., Phys. Rev. D 74, 022001 (2006).

[14] V. B. Braginsky and F. Ya. Khalili, Phys. Lett. A 257, 241 (1999).

[15] V. B. Braginsky, M. L. Gorodetsky, and F. Y. Khalili, Phys. Lett. A 232, 340 (1997).

[16] H. Rehbein, H. Mueller-Ebhardt, K. Somiya, C. Li, R. Schnabel, K. Danzmann, and Y. Chen, Phys. Rev. D 76, 062002 (2007).

[17] E. Gustafson, D. Shoemaker, K. Strain, and R. Weiss, Report No. LIGO-T990080-00-D, 1999 (http://www.ligo. caltech.edu/docs/T/T990080-00.pdf).

[18] T. Corbitt, Y. Chen, F. Khalili, D. Ottaway, S. Vyatchanin, S. Whitcomb, and N. Mavalvala, Phys. Rev. A 73, 023801 (2006).

[19] H. Mueller-Ebhardt, H. Rehbein, T. Corbitt, C. Wipf, N. Mavalvala, R. Schnabel, K. Danzmann, and Y. Chen (unpublished).

[20] T. Corbitt, Y. Chen, E. Innerhofer, H. Mueller-Ebhardt, D. Ottaway, H. Rehbein, D. Sigg, S. Whitcomb, C. Wipf, and N. Mavalvala, Phys. Rev. Lett. 98, 150802 (2007).

[21] A. Schliesser, P. Del'Haye, N. Nooshi, K. J. Vahala, and
T. J. Kippenberg, Phys. Rev. Lett. 97, 243905 (2006).

[22] H. J. Kimble, Y. Levin, A. B. Matsko, K. S. Thorne, and S. P. Vyatchanin, Phys. Rev. D 65, 022002 (2001).

[23] T. Corbitt, Y. Chen, and N. Mavalvala, Phys. Rev. A 72, 013818 (2005).

[24] J. Harms, R. Schnabel, and K. Danzmann, Phys. Rev. D 70, 102001 (2004).

[25] V.B. Braginsky and F. Y. Khalili, Quantum Measurement (Cambridge University Press, Cambridge, England, 1999).

[26] http://www.ligo.mit.edu/bench/bench.html.

[27] S. A. Hughes and K. S. Thorne, Phys. Rev. D 58, 122002 (1998).

[28] R. Adhikari (private communication).

[29] A. Bunkowski, O. Burmeister, D. Friedrich, K. Danzmann, and R. Schnabel, Classical Quantum Gravity 23, 7297 (2006).

[30] M. Bondarescu and K. S. Thorne, Phys. Rev. D 74, 082003 (2006).

[31] T. Uchiyama et al., Phys. Lett. A 261, 5 (1999).

[32] T. Uchiyama, D. Tatsumi, T. Tomaru, M.E. Tobar, K. Kuroda, T. Suzuki, N. Sato, A. Yamamoto, T. Haruyama, and T. Shintomi, Phys. Lett. A 242, 211 (1998).

[33] K. McKenzie, N. Grosse, W. P. Bowen, S. E. Whitcomb, M. B. Gray, D. E. McClelland, and P. K. Lam, Phys. Rev. Lett. 93, 161105 (2004).

[34] H. Vahlbruch, S. Chelkowski, B. Hage, A. Franzen, K. Danzmann, and R. Schnabel, Phys. Rev. Lett. 97, 011101 (2006).

[35] J. Harms, Y. Chen, S. Chelkowski, A. Franzen, H. Vahlbruch, K. Danzmann, and R. Schnabel, Phys. Rev. D 68, 042001 (2003).

[36] A. Buonanno and Y. Chen, Phys. Rev. D 69, 102004 (2004).

[37] H. Vahlbruch, S. Chelkowski, B. Hage, A. Franzen, K. Danzmann, and R. Schnabel, Phys. Rev. Lett. 95, 211102 (2005). 